Ocular Oncology

and Pathology
Ocul Oncol Pathol 2017;3:199-203

DOI: $10.1159 / 000454863$
Received: September 30,2016

Accepted after revision: November 25, 2016

Published online: February 15, 2017

\title{
Ciliary Body Mesectodermal Leiomyoma Diagnosed by Fine Needle Aspiration Biopsy
}

\author{
Hayyam Kiratlia Abdullah Ağın ${ }^{a}$ Sevgen Önder ${ }^{b}$ \\ ${ }^{a}$ Ocular Oncology Service, Department of Ophthalmology, and ${ }^{b}$ Department of Pathology, Hacettepe University, \\ School of Medicine, Ankara, Turkey
}

\section{Established Facts}

- Mesectodermal leiomyoma is a rare ciliary body tumor arising from the neural crest-derived ciliary muscle.

- Removal of the tumor or the eye continues to be the only option for definitive diagnosis and treatment.

\section{Novel Insights}

- Ciliary body mesectodermal leiomyoma can be diagnosed with transscleral fine needle aspiration biopsy.

- Stereotactic radiotherapy can reduce the size of the tumor slowly over the years.

\section{Keywords}

Mesectodermal leiomyoma · Ciliary body · Fine needle aspiration · Cytology · Radiotherapy

\begin{abstract}
Aims: To describe the clinical, imaging, cytopathological, and immunohistochemical features of mesectodermal leiomyoma of the ciliary body diagnosed by fine needle aspiration biopsy in a patient who denied any major intervention without prior pathological verification. Methods: The clinical, imaging, and cytopathological records of the patient were retrospectively reviewed. A fine needle aspiration biopsy with a $25-G$ needle was performed after tailoring a scleral flap. Results: Examination of the cell blocks prepared
\end{abstract}

\section{KARGER}

(c) 2017 S. Karger AG, Basel

E-Mail karger@karger.com

www.karger.com/oop from the aspirate demonstrated cells with granular cytoplasms and large nuclei. Immunohistochemical studies showed positive smooth muscle actin, desmin, h-caldesmon, CD56, and neuron-specific enolase stainings, suggesting both neurogenic and myogenic differentiation. Reactions for HMB-45, S-100, panCK, epithelial membrane antigen, glial fibrillary acidic protein, and CD68 were negative. Because the tumor continued to enlarge, fractionated stereotactic radiotherapy at the dose of 3,000 cGy was delivered, which reduced the size of the mass by $40 \%$ in 5 years. Conclusions: This case demonstrated that the diagnosis of mesectodermal leiomyoma of the ciliary body can be established with fine needle aspiration biopsy and that stereotactic radiotherapy can be successfully used to induce tumor regression.

(c) 2017 S. Karger AG, Basel
Hayyam Kiratli, MD

Professor of Ophthalmology, Ocular Oncology Service

Hacettepe University School of Medicine

Sihhiye, TR-06100 Ankara (Turkey)

E-Mail hkiratli@hacettepe.edu.tr 


\section{Introduction}

Intraocular leiomyoma is a rare, benign tumor arising most commonly from the ciliary body $(70 \%)$ followed by the iris $(25 \%)[1,2]$. Strictly unilateral, this tumor is more often encountered in young adult females between 20-50 years of age [1-3]. A ciliary body leiomyoma usually presents as a well-circumscribed, melanocytic (55\% of cases) retrolenticular mass with prominent intrinsic vasculature, inducing astigmatism and having episcleral sentinel vessels [1-5]. These nonspecific signs and currently available imaging modalities do not allow to distinguish leiomyoma precisely from the more common melanoma and other rare tumors including adenoma of the ciliary epithelium, schwannoma, metastatic melanoma, or neurofibroma $[2,3]$. The diagnoses in the great majority of previously well-documented cases were based on histopathological and/or ultrastructural examination of either enucleation or resected tumor specimens $[6,7]$.

We describe the case of a young woman with ciliary body mesectodermal leiomyoma through which we aim to emphasize the determinant contribution of fine needle aspiration biopsy in the final diagnosis and also the role of stereotactic radiotherapy in the management of this rare tumor.

\section{Case Report}

In 2010, an otherwise healthy 24-year-old woman presented with the complaint of gradual visual loss in her left eye, which developed in 3 months. Her visual acuity was 20/20 in the right eye and 20/200 in the left eye. Biomicroscopic examination revealed a partially transilluminating amelanotic ciliary body mass in the temporal part of the left eye causing iridodialysis and nasal displacement of the lens (Fig. 1). There was no cellular reaction in the anterior chamber and vitreous. Her intraocular pressures were 12 $\mathrm{mm} \mathrm{Hg}$ in the right eye and $18 \mathrm{~mm} \mathrm{Hg}$ in the left eye. The right eye was normal. Orbital magnetic resonance imaging (MRI) studies demonstrated a well-delineated ciliary body mass with subluxation of the lens (Fig. 2). The patient was offered enucleation, transscleral tumor resection, or stereotactic radiotherapy as treatment options with the presumptive diagnosis of an amelanotic melanoma. However, the patient requested tissue diagnosis before proceeding to any treatment and we performed transscleral fine needle aspiration biopsy with a $25-\mathrm{G}$ needle after dissection of a small square scleral flap. Examination of the paraffin-embedded cell block prepared from the aspirate disclosed cells with thin granular cytoplasm, oval-round nuclei, and prominent nucleoli. Distinctive cytologic atypia, mitosis, or necrosis were not detected. Immunohistochemical studies showed strong positive staining with smooth muscle differentiation markers including smooth muscle-specific actin (SMA), desmin, and h-caldesmon, as well as neuronal differentiation marker CD56 (Fig. 3). Weak positive reaction for neu-

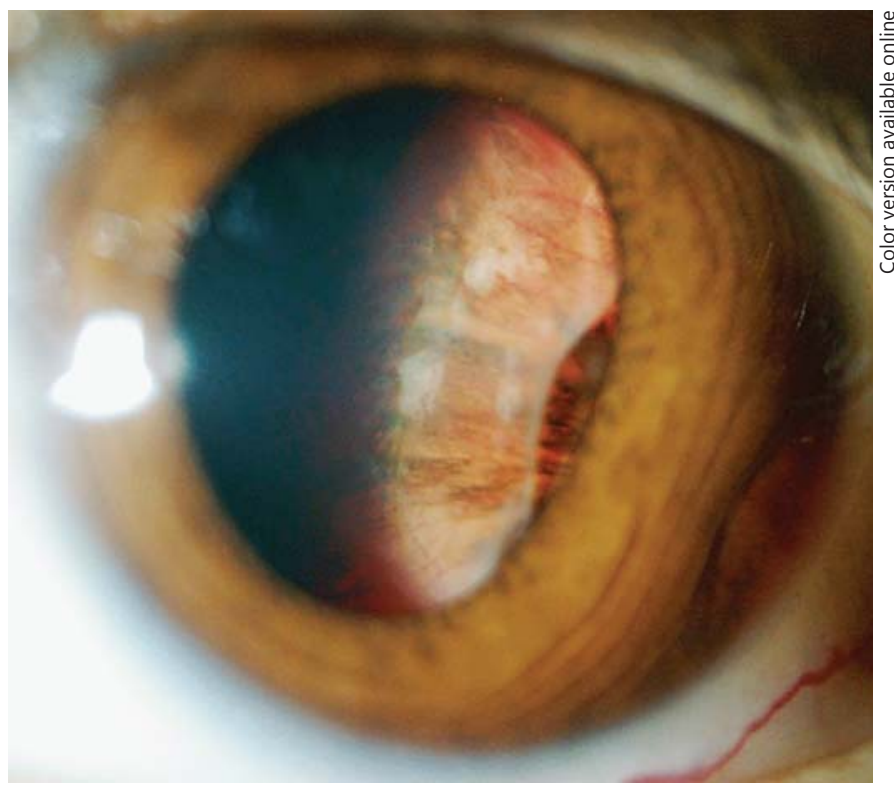

Fig. 1. Slit-lamp examination showed an amelanotic tumor with temporal iridodialysis which partially transmitted light.

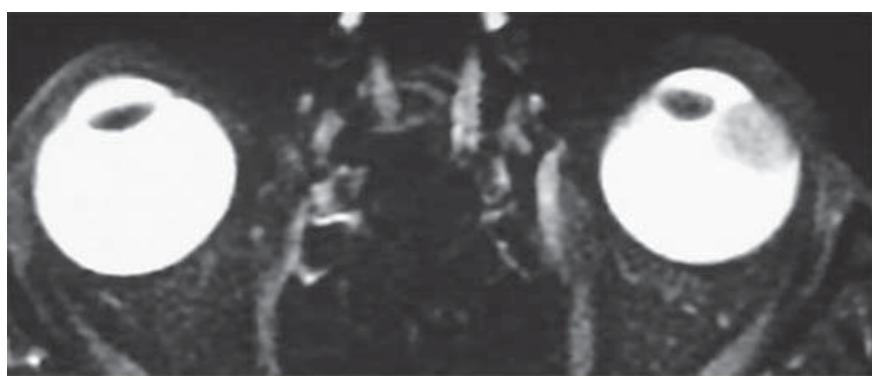

Fig. 2. T2-weighted axial orbital MRI scan showed a hypointense ciliary body mass on the temporal part of the left eye, causing subluxation of the lens.

ron-specific enolase was also observed. Immunostains for HMB45, S-100, panCK, epithelial membrane antigen, estrogen and androgen receptors, GFAP, and CD68 were negative. The Ki-67 index was less than $1 \%$. These findings suggested mesectodermal leiomyoma with neuronal differentiation and allowed us to rule out ciliary body melanoma. The patient refused treatment for another year until further tumor growth and displacement of the lens were documented (Fig. 4). This time, a shallow serous retinal detachment was present posterior to the tumor and involved the macula. She then consented for radiotherapy which was delivered in the form of stereotactic radiotherapy prescribing 3,000 cGy in 20 fractions to the tumor. Two years later, she developed cataract, which was removed with standard extracapsular technique without intraocular lens implantation. During 5 years of follow-up, the tumor regressed for about $40 \%$ with total resorption of the subretinal fluid (Fig. 5). Her visual acuity in the left eye was 20/80 with contact lens correction at the writing of this report. 

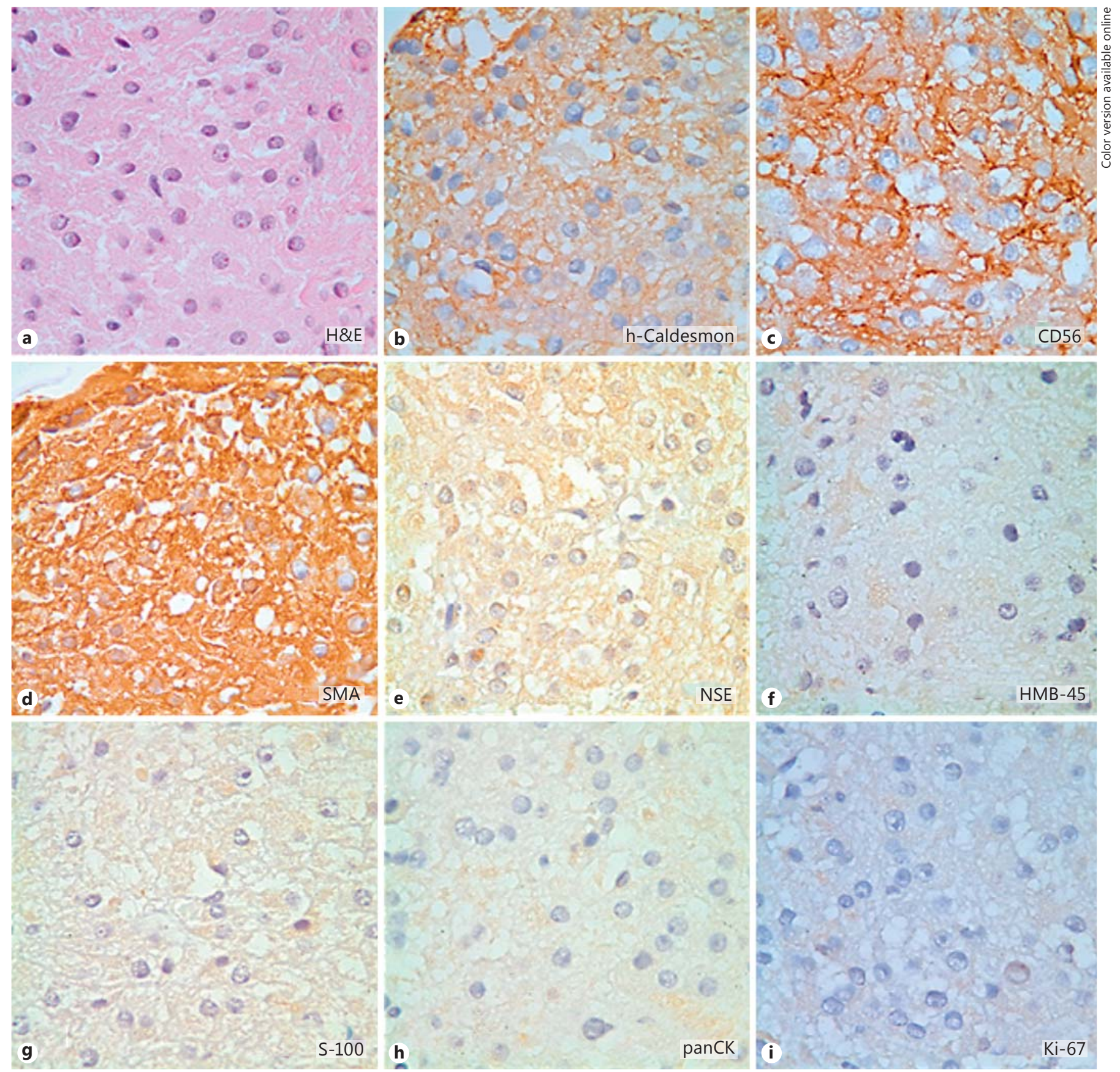

Fig. 3. Immunohistopathological evaluation demonstrated tumor cells with large, finely granular cytoplasms and oval nuclei (H\&E, $\times 80$; a), strong positive expressions of h-caldesmon (b), CD56 (c), and smooth muscle actin (SMA; d), weak expression of neuron-specific enolase (NSE; e), and negative staining for HMB-45 (f), S-100 (g), and panCK $(\mathbf{h})$. The Ki-67 index was $<1 \%(\mathbf{i})$.

\section{Discussion}

Intraocular leiomyoma may arise either from the vascular smooth muscles, which are derived from the meso- derm or from the ciliary muscles, which are of neural crest origin [2-4]. The latter is termed mesectodermal leiomyoma and is distinguished from the conventional leiomyoma by the presence of both myogenic and neuro- 


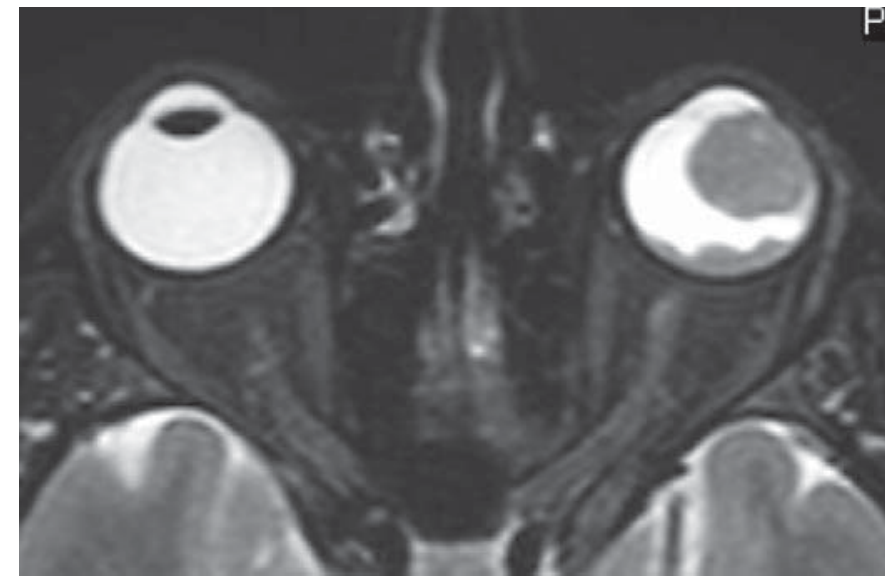

Fig. 4. T2-weighted axial orbital MRI scan documented the growth of the tumor with accompanying exudative retinal detachment a year after the diagnosis.

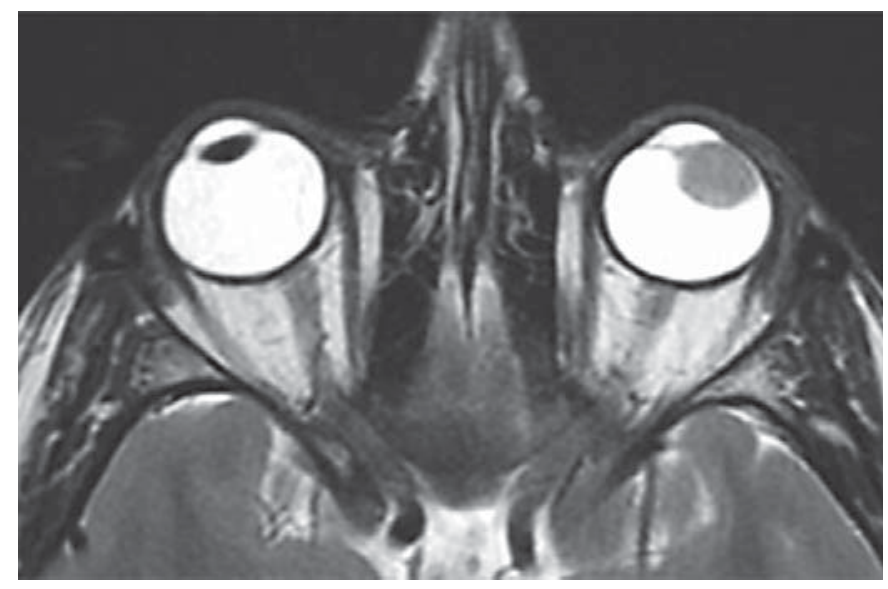

Fig. 5. This T2-weighted axial orbital MRI scan showed a measurable tumor regression 5 years after stereotactic radiotherapy without any radiological evidence of retinal detachment.

genic histologic features [8]. A recent literature review identified only 25 well-documented cases of ciliary body mesectodermal leiomyomas which were diagnosed after enucleation or resection of the whole tumor [7]. Our case differs from most previously reported cases in two aspects.

First, the diagnosis in our case was established with fine needle aspiration biopsy. The demonstration of negative results for HMB-45 and S-100 confidently ruled out a melanoma. Similar to other reported cases, expressions of desmin, SMA, and caldesmon were suggestive of a leiomyoma in our patient $[3,9,10]$. There seems to be no difference between mesodermal and mesectodermal leiomyomas regarding clinical presentation, imaging characteristics, and prognosis. However, at histopathological level, mesectodermal leiomyomas may be more vascular and appear paucicellular compared to conventional leiomyomas [1]. More importantly, the strong expression of CD56, which is a neural marker, and a weak positivity for neuron-specific enolase weighed in favor of mesectodermal leiomyoma in our case. All these detailed evaluations were performed on cell blocks obtained with fine needle aspiration biopsy. We are aware of only one other patient in whom fine needle aspiration biopsy from a ciliary body tumor clearly suggested the diagnosis of mesectodermal leiomyoma [11]. This was archival material from the University of California, Los Angeles and the aspirate showed clusters of oval and spindle cells with bland and inconspicuous nucleoli in a fibrillary background [11]. Histopathological sections available from the same case demonstrated a highly vascular tumor with a myxoid and fibrillary background with neuronal features, and it was by electron microscopy that smooth muscle differentiation was identified [11].

A recent study showed expressions of androgen and progesterone receptors in 3 patients with mesectodermal leiomyoma, awaiting further confirmation [12]. In our case, estrogen and androgen receptors were not expressed in examined cell blocks.

The second unconventional aspect of our case was the partial response to stereotactic radiotherapy. All previous reports had the main purpose of demonstrating convincingly certain distinguishing histopathological and immunohistochemical features of mesectodermal leiomyoma in patients who were treated with enucleation or tumor resection $[1,4-7,9,10]$. We were unable to find a patient with mesectodermal leiomyoma in the literature treated with radiotherapy. Theoretically, since leiomyoma is a benign, slow growing tumor, the effect of radiotherapy would be expected to be limited at best. However, our experience with this patient suggested that the irradiation dose of 30 Gy delivered in fractions resulted in $40 \%$ reduction in tumor size over 5 years. In conclusion, mesectodermal leiomyoma of the ciliary body can be diagnosed with fine needle aspiration biopsy and managed conservatively with radiotherapy. Long-term follow-up is necessary for any conclusion to be drawn regarding the efficacy of radiotherapy. 


\section{Statement of Ethics}

Informed consent was obtained from the patient to utilize all available medical records for publication and other educational purposes.

\section{Disclosure Statement}

The authors have no conflicts of interest. The authors did not receive any funding or sponsorship for the preparation of the manuscript.

\section{References}

1 Shields JA, Shields CL, Eagle RC, De Potter P: Observation of seven cases of intraocular leiomyoma. The 1993 Byron Demorest lecture. Arch Ophthalmol 1994;112:521-528.

2 Rundle P, Mudhar HS: Uveal myogenic, fibrous and histiocytic tumors; in Damato B, Singh AD (eds): Clinical Ophthalmic Oncology. Berlin/Heidelberg, Springer, 2014, pp 331-339.

3 Lai CT, Tai MC, Liang CM, Lee HS: Unusual uveal tract tumor: mesectodermal leiomyoma of the ciliary body. Pathol Int 2004;54:337342.

4 Biswas J, Kumar K, Gopal L, Bhende MP: Leiomyoma of the ciliary body extending to the anterior chamber: clinicopathologic and ultrasound biomicroscopic correlation. Surv Ophthalmol 2000;44:336-342.
5 Kiratli H, Bilgiç S, Söylemezoğlu F: Léiomyome du corps ciliaire. A propos de 3 cas. J Fr Ophtalmol 2005;28:1105-1109.

6 Ishigooka H, Yamabe H, Kobashi Y, Nagata $\mathrm{M}$ : Clinical and pathological status of mesectodermal leiomyoma of the ciliary body. A case report and review of the literature. Graefes Arch Clin Exp Ophthalmol 1989;227:101105.

7 Renteria-Ruiz NP, de Witt-Carter G, Villasenor-Diez J, Rodriguez-Reyes AA: Leiomioma mesectodérmico. Un tumor poco frecuente del cuerpo ciliar. Cirurgia y Cirujanos 2014; 82:70-75.

8 Jakobiec FA, Font RL, Tso MOM, Zimmerman LE: Mesectodermal leiomyoma of the ciliary body. A tumor of presumed neural crest origin. Cancer 1977;39:2102-2113.
9 Odashiro AN, Fernandes BF, Al Kandari A, Gregoire FJ, Burnier MN: Report of two cases of ciliary body mesectodermal leiomyoma: unique expression of neural markers. Ophthalmology 2007;114:157-161.

10 Koletsa T, Karayannopoulou G, Dereklis D, Vasileaidis I, Papadimitriou C, Hytiroglou P: Mesectodermal leiomyoma of the ciliary body: report of a case and review of the literature. Pathol Res Pract 2009;205:125-130.

11 Glasgow BJ, Foos RY: Ocular Cytopathology. Oxford, Butterworth-Heinemann, 1993, pp 43-47.

12 Quhill H, Rennie IG, Rundle PA, Mudhar HS Three cases of intraocular mesectodermal leiomyoma expressing progesterone and androgen receptors. Eye 2013;27:669-672. 